\title{
Short cavity InGaAsP/InP lasers with dielectric mirrors
}

\author{
U. Koren, Z. Rav-Noy, A. Hasson, T. R. Chen, K. L. Yu, L. C. Chiu, S. Margalit, and \\ A. Yariv \\ California Institute of Technology, Pasadena, California 91125
}

(Received 13 December 1982; accepted for publication 1 February 1983)

\begin{abstract}
Short cavity length $(38 \mu \mathrm{m})$ lasers have been fabricated using a recently developed microcleavage technique. $\mathrm{SiO}_{2}$-amorphous $\mathrm{Si}$ multilayer coatings have been evaported on the lasers to obtain high reflectivity mirrors. The lasers have current thresholds as low as $3.8 \mathrm{~mA}$ with $85 \%$ reflecting front mirror and high reflectivity rear mirror and $2.9 \mathrm{~mA}$ with two high reflectivity mirrors. Single longitudinal mode operation is observed over a wide range of driving currents and temperatures.
\end{abstract}

PACS numbers: 42.55.Px

Short cavity injection lasers have been investigated in the InGaAsP/InP ${ }^{1,2}$ and in the GaAlAs/ GaAs $s^{3,4}$ material systems. It has been demonstrated theoretically ${ }^{1}$ and experimentally ${ }^{1-3}$ that short cavity lasers (with $30-70 \mu \mathrm{m}$ cavity length) provide consistent single longitudinal mode operation over wide current and temperature ranges. It has also been observed and theoretically explained ${ }^{1}$ that high reflectivity mirrors contribute to the single mode properties of these lasers.

Here, we report the fabrication and properties of short cavity lasers obtained by microcleavage. ${ }^{3,5,6}$ This technique makes it possible to obtain high quality mirrors by cleaving small suspended bridge structures that are fabricated on the wafer. After microcleavage, dielectric multilayer coatings are deposited by evaporation from two directions so that high reflectivity mirrors are obtained for both sides of each of the lasers on the wafer. The use of dielectric multilayer mirrors is advantageous for accurate control over mirror reflectivities and for very high reflectivity mirrors. Also, these mirrors are potentially highly resistant to damage at high power densities due to the low optical absorption and high quality of the mirror materials.

The external differential quantum efficiency obtained for lasers with $85 \%$ reflectivity for the front mirror and highly reflecting rear mirror is of the orderof $20 \%$ with most of the power emerging from the front mirror. This is comparable to the performance of conventional long cavity lasers. However, the reduction of mirror losses due to the highly reflecting mirrors also causes a significant drop in the threshold currents resulting in very low threshold lasers. Another advantage is that this configuration provides good heat sinking, since the short laser is located on a bigger substrate and heat is dissipated with a point source geometry to the substrate. This makes it possible to have cw operation of the lasers at high $I / I_{\text {th }}$ values.

As demonstrated by previous work, our results also show that short cavity lasers with high reflectivity mirrors have a strong preference for single longitudinal mode operation. It is also possible, however, to obtain stable dual mode operation of these lasers when the gain peak is located near the midpoint between two consequent longitudinal modes. ${ }^{1}$ This may be useful for applications where the beat frequency of the two modes is of interest. Stable dual mode operation can be obtained by fine temperature tuning and it has been observed here by time-resolved pulsed spectral measurements.

The schematic diagram of the device is shown in Fig. 1(a) and a top view photomicrograph of the complete device is shown in Fig. 1(b). The laser structure and microcleavage technique have been reported recently. ${ }^{5}$ Here, the same laser is fabricated with $38-\mu \mathrm{m}$ cavity length and with the negative contact located on a larger mesa at the side of the laser channel.

After microcleavage, the reflective multilayer coatings are evaporated using an electron beam gun evaporation system. The wafer is mounted on a two-stage rotational station

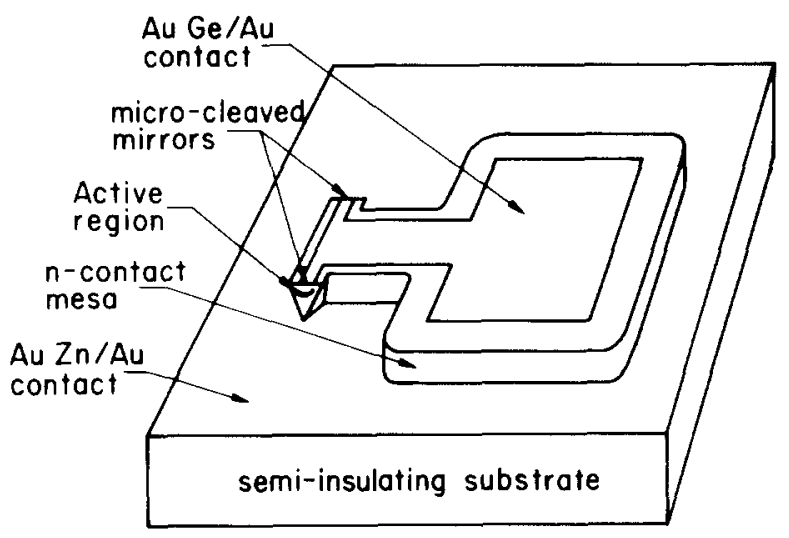

(a)

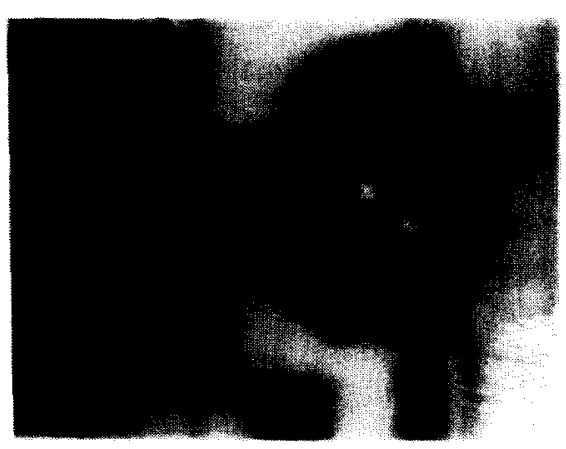

(b)

$$
\overleftrightarrow{20 \mu m}
$$

FIG. 1. (a) Schematic structure of the short cavity laser. (b) Top view photomicrograph of the complete device. 


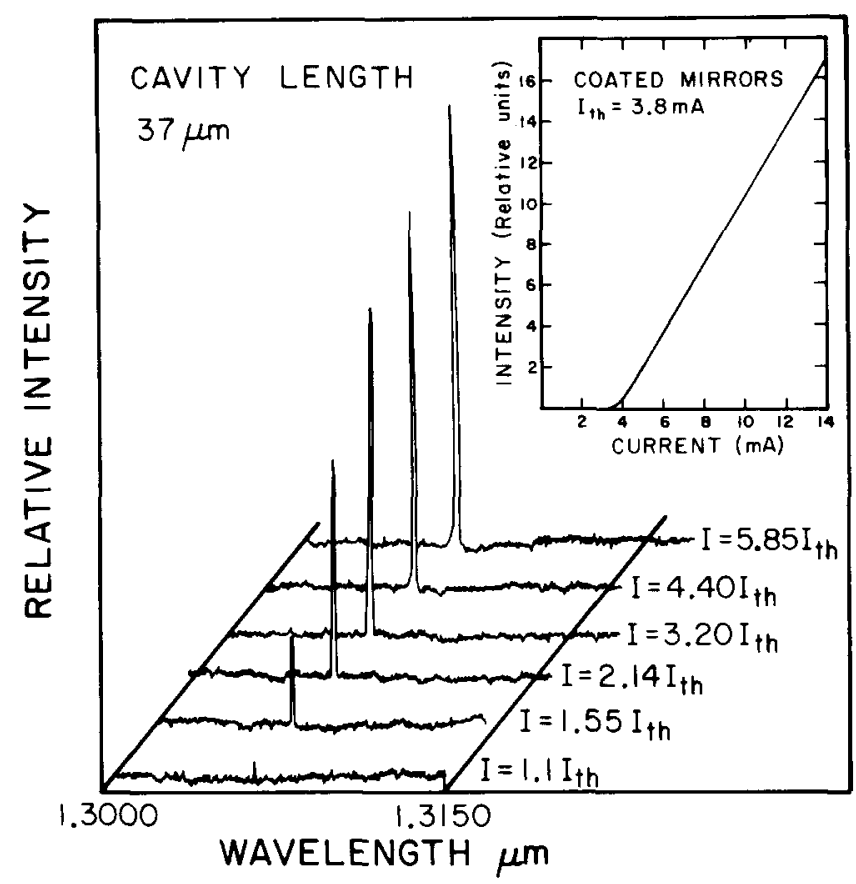

FIG. 2. Spectral behavior of $37-\mu \mathrm{m}$ cavity length laser with front mirror$85 \%$ relfectivity, rear mirror-high reflectivity. The insert shows the lightcurrent characterisitics of a similar laser with $38-\mu \mathrm{m}$ cavity length.

inside the vacuum system. The mirrors are coated with $10^{\circ}$ inclination between the normal to the mirrors and the direction of evaporation. Following evaporation of the first mirror, the wafer is rotated $180^{\circ}$ and evaporation on the mirrors of the other side of the lasers is performed. The coatings are made of alternating $\mathrm{SiO}_{2}$ and amorphous $\mathrm{Si}$ layers of $1 / 4 \lambda$ thickness with the last layer being of $1 / 8 \lambda \mathrm{Si}$. The $\mathrm{SiO}_{2}$ is deposited from a fused silica source and the $\mathrm{Si}$ is evaporated from a single crystal high purity $\mathrm{Si}$. The reflection at normal incidence for $1.3-\mu \mathrm{m}$ wavelength is $98.5 \%$ and $85 \%$ for the rear and front mirrors, respectively, with the mirrors deposited on glass substrates. The front and rear mirrors are composed of 6 and 10 layers, respectively.

Light-current characteristics of a $38-\mu \mathrm{m}$ cavity length laser with $85 \%$ reflective front mirror are shown in the insert of Fig. 2. The lowest threshold current obtained is $3.8 \mathrm{~mA}$.

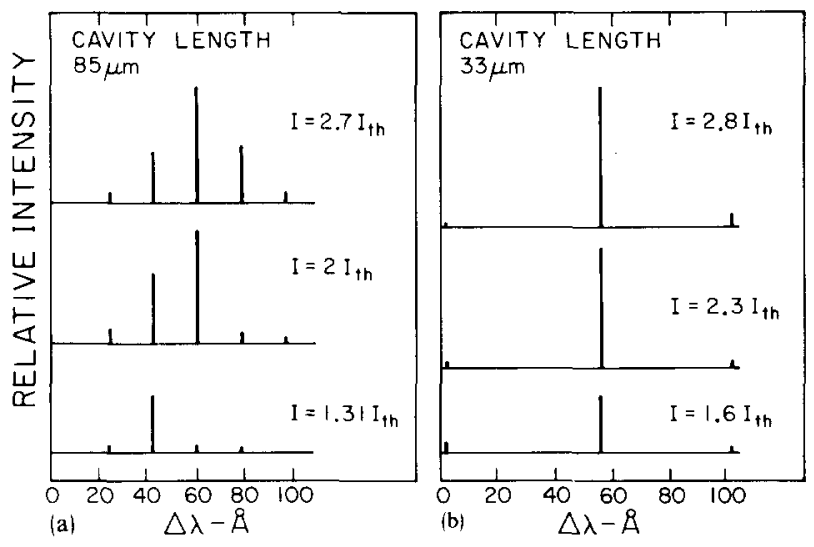

FIG. 3. (a) Spectral behavior of $85-\mu \mathrm{m}$ cavity length laser with uncoated mirrors. (b) Spectral behavior of a laser with $33-\mu \mathrm{m}$ cavity length with uncoated mirrors.

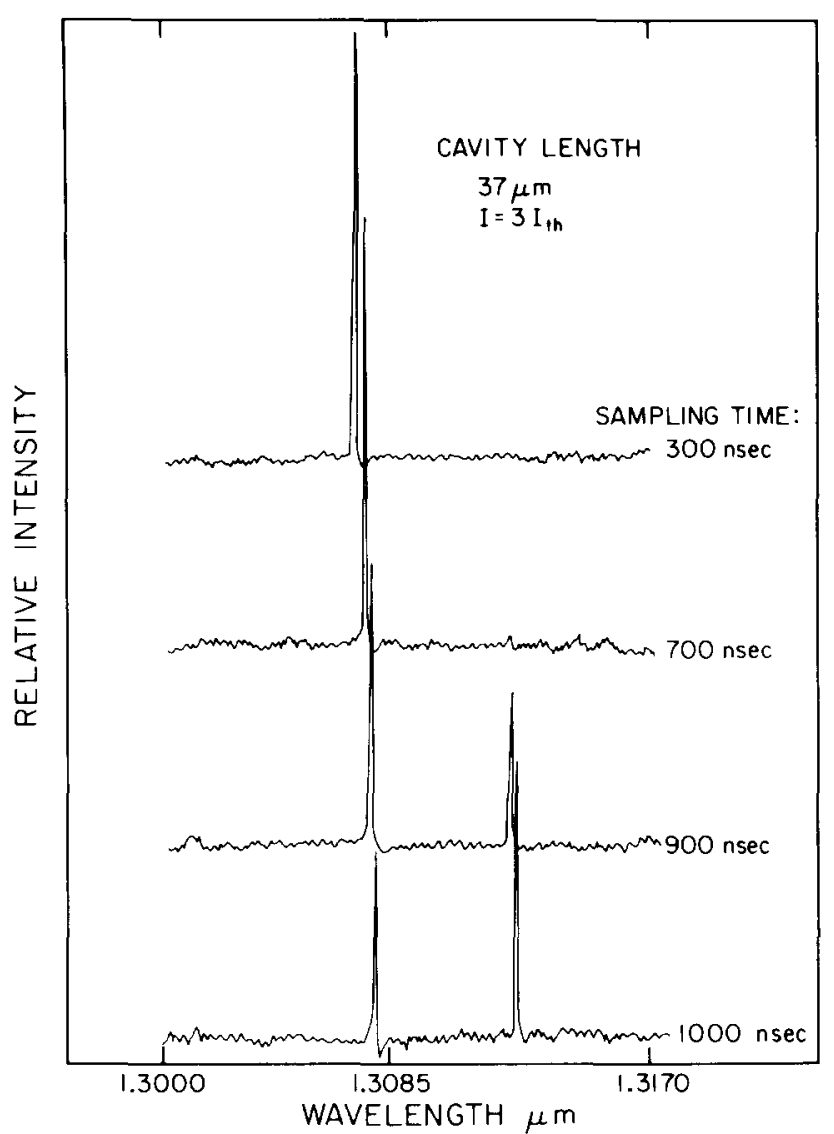

FIG. 4. Time-resolved pulsed spectral behavior of a $37-\mu \mathrm{m}$ cavity length laser with $85 \%$ reflective front mirror and high reflectivity rear mirror, pulse duration $1 \mu \mathrm{s}$, repetition rate $-3 \mathbf{K H}_{3}$, sampling window $-1 \mathrm{~ns}$.

The threshold current of the same device without reflective coatings is $22 \mathrm{~mA}$. With high reflectivity 10 layers front mirror and 14 layers rear mirror, the threshold current is 2.9 $\mathrm{mA}$, but the quantum efficiency is very low. With these results and the known approximate mirror reflectivities, one can estimate the internal cavity loss to be of the order of 40$50 \mathrm{~cm}^{-1}$, and the mirror loss (for the $85 \%$ front mirror laser) is $\sim 25 \mathrm{~cm}^{-1}$. The observed external differential quantum efficiency is about $20 \%$, and the power coming out of the front mirror is higher by a factor of 5-7 than the power emerging from the rear mirror.

The voltage drop on the lasers at threshold is as low as $1.3-1.5 \mathrm{~V}$. cw operation is obtained at the same threshold current as for pulsed operation. cw operation at up to $5 I_{\text {th }}$ has been obtained for the low threshold devices without strong heating of the lasers.

The spectral behavior of the short cavity lasers with uncoated mirrors is shown in Fig. 3. For $85-\mu \mathrm{m}$ cavity length [Fig. 3(a)] the laser shows multimode operation at currents higher than $1.5 I_{\mathrm{th}}$. For 33- $\mu \mathrm{m}$ cavity length [Fig. 3(b)] the laser operates at a single mode, but there is a noticeable power at the next order secondary modes. The spectral response of a $37-\mu \mathrm{m}$ laser with coated mirrors $(85 \%$ reflective front mirror) is shown in Fig. 2 for 200 -ns current pulses. Single mode operation is maintained at up to $6 I_{\text {th }}$ with no noticeable power at secondary modes.

Although this laser shows strong single mode behavior, it is also possible by fine temperature tuning to stabilize it at 
dual mode operation. This is demonstrated by the time-resolved 1- $\mu$ s pulsed spectral measurements shown in Fig. 4. During the on time of square current pulse (with $I=3 I_{\mathrm{th}}$ ), the temperature is increasing continuously and the gain peak shifts to the right relatively to the modes position. Thus, at the temporal window between $900-1000$ ns dual mode operation is observed.

In conclusion, short cavity lasers have been fabricated using microcleavage techniques. High reflectivity multilayer dielectric coatings have been evaporated on the laser mirrors. The lasers show very low threshold cw operation with external quantum efficiency that is comarable to conventiaonl long cavity lasers. The lasers operate at a single longitudinal mode over wide ranges of driving currents and temperatures.
This work was supported by the Office of Naval Research and the Air Force Office of Scientific Research.

'T. P. Lee, C. A. Burrus, J. A. Copeland, A. G. Denati, and D. Marcuse, J. Quantum. Electron. QE-18 1101 (1982).

${ }^{2}$ C.A. Burrus, T. P. Lee, and A. G. Denati, Electron. Lett. 17, 954 (1981). ${ }^{3}$ B. F. Levine, J. P. Van der ziel, and R. A. Logan, "Low threshold microcleaved (GaAl)As lasers," 8th IEEE International Semiconductor Laser Conference, September, 1982, paper No. 16.

${ }^{4}$ N. Bouadma, J. Rion, and J. C. Bouley, Electron. Lett. 18, 879 (1982). ${ }^{5}$ H. Blauvelt, N. Bar-chaim, D. Fekete, S. Margalit, and A. Yariv, Appl. Phys. Lett. 40, 289 (1982).

${ }^{6}$ U. Koren, A. Hasson, K. L. Yu, T. R. Chen, S. Margalit, and A. Yariv, Appl. Phys. Lett. 41, 791 (1982)

\title{
New large optical cavity laser with distributed active layers
}

\author{
W. T. Tsang and N. A. Olsson \\ Bell Laboratories, Murray Hill, New Jersey 07974
}

(Received 28 October 1982; accepted for publication 13 January 1983)

\begin{abstract}
A new semiconductor current injection laser having multiple active layers distributed within a large optical cavity (LOC) waveguide is shown to operate as an efficient, low-threshold, high-peak power laser diode. The use of distributed active layers in LOC laser structure leads to improved efficiency in utilizing the injected carriers when compared to the conventional single active layer LOC laser structure.
\end{abstract}

PACS numbers: $42.55 . \mathrm{Px}, 42.80 . \mathrm{Sa}, 68.55 .+\mathrm{b}$

Previously, high-power devices consisting of a stack of individual single heterostructure $\mathrm{GaAs} / \mathrm{Al}_{x} \mathrm{Ga}_{1-x} \mathrm{As}$ laser chips soldered together have been developed. ${ }^{1}$ Recently, an integrated version of these devices with the crystal tunnel junctions replacing the solder joints was demonstrated. ${ }^{2}$ In both cases, the light-emitting active layers have to be separated far apart in order to avoid material absorption by the metallic joints in the former or by the $p^{+} n^{+}$GaAs tunnel junctions in the latter. As a result, the optical outputs from the lasers in the stack are not coupled and hence incoherent from each other. Furthermore, in order for all the individual lasers to lase simultaneously and the device to have linear light-current characteristic, all the lasers have to be identical. ${ }^{2}$ Such requirement is quite stringent for the former. ${ }^{\prime}$

In this letter we propose and demonstrate the operation of a new semiconductor current injection heterostructure laser: the large optical cavity distributed active layer (LOCDAL) laser, as an alternative to the above approaches, ${ }^{1.2}$ for high-power applications. Figure 1(a) shows a schematic diagram of the cross section of the LOC-DAL laser, while Fig. 1 (b) shows the scanning electron microscopy photograph of such a device with four active layers evenly distributed within the LOC. The spacings and thicknesses of the active layers in the LOC can also be made different if desired. Unlike the conventional LOC lasers first proposed by Lockwood et al. ${ }^{3}$ in which only a single active layer is incorporated adjacent to the large optical cavity waveguide, there are multiple active layers distributed within the LOC waveguide in the present structure. In the framework of the previous stacked lasers, ${ }^{1,2}$ this can be viewed as having a series of double heterostructures (DH) closely stacked on top of each other and sandwiched between two outer cladding layers containing a $p-n$ junction. It can also be considered as having the barrier layer thickness in the modified multiquantum well (MQW) heterostructure lasers ${ }^{4}$ increased from $\leqslant 200 \AA$ to $\gtrsim 2000 \AA$ so as to form a LOC in order to increase the output power capability. Uniform injection of carriers into the various $\mathrm{Al}_{x} \mathrm{Ga}_{1-x}$ As active layers is achieved by having the AlAs composition in the $\mathrm{Al}_{y} \mathrm{Ga}_{1-y} \mathrm{As} \mathrm{LOC}$ waveguide $(y-x) \leqslant 0.2$ and the total thickness of the LOC smaller than the diffusion lengths of the carriers, $\approx 3 \mu \mathrm{m}^{4}$ With this structure only a single build-in $p-n$ junction is employed to inject carriers into all the active layers. Since the active layers are made $\leqslant 1000 \AA$ and are spaced by $\mathrm{Al}_{y} \mathrm{Ga}_{1-y}$ As layers of $\lesssim 3500 \AA$, they are tightly coupled so that the entire LOC with the DAL forms a single waveguide. The large thickness of the LOC significantly increases the maximum output power possible before the threshold optical density for catastrophic damage at the cleaved mirror occurs which is between 3 and $5 \mathrm{MW} / \mathrm{cm}^{2}$.

Two such structures were grown by molecular beam epitaxy (MBE). One with four GaAs active layers, each of $700 \AA$ and separated by $\mathrm{Al}_{0.1} \mathrm{Ga}_{0.9}$ As of $3200 \AA$ with a total LOC-DAL thickness of $1.24 \mu \mathrm{m}$ (wafer a). The other has six 